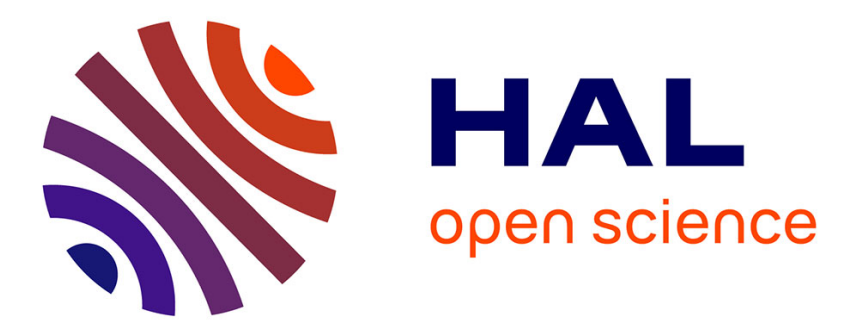

\title{
La force de la forme. Discipline et socialisation des jeunes sans diplôme en internat d'inspiration militaire
}

Joël Zaffran

\section{To cite this version:}

Joël Zaffran. La force de la forme. Discipline et socialisation des jeunes sans diplôme en internat d'inspiration militaire. Formation Emploi. Revue française de sciences sociales, 2019, 148, pp.47-67. 10.4000/formationemploi.7800 . halshs-02418085

\section{HAL Id: halshs-02418085 \\ https://shs.hal.science/halshs-02418085}

Submitted on 4 Jan 2022

HAL is a multi-disciplinary open access archive for the deposit and dissemination of scientific research documents, whether they are published or not. The documents may come from teaching and research institutions in France or abroad, or from public or private research centers.
L'archive ouverte pluridisciplinaire HAL, est destinée au dépôt et à la diffusion de documents scientifiques de niveau recherche, publiés ou non, émanant des établissements d'enseignement et de recherche français ou étrangers, des laboratoires publics ou privés. 
FORMATION Formation emploi

Revue française de sciences sociales

148 | Octobre-Décembre

Varia

\section{La force de la forme.Discipline et socialisation des jeunes sans diplôme en internat d'inspiration militaire}

The strength of the form. Discipline and socialization of young people without diploma in military inspiration residency.

Die Macht der Form. Disziplin und Sozialisierung von Jugendlichen ohne abgeschlossene Ausbildung in Internaten mit militärischem Charakter La fuerza de la forma. Disciplina y socialización de los jóvenes sin estudios en internados de inspiración militar

Joël Zaffran

\section{OpenEdition}

Journals

Édition électronique

URL : http://journals.openedition.org/formationemploi/7800

DOI : 10.4000/formationemploi.7800

ISSN : 2107-0946

Éditeur

La Documentation française

Édition imprimée

Date de publication : 31 décembre 2019

Pagination : 47-67

ISSN : 0759-6340

Référence électronique

Joël Zaffran, « La force de la forme.Discipline et socialisation des jeunes sans diplôme en internat d'inspiration militaire », Formation emploi [En ligne], 148 | Octobre-Décembre, mis en ligne le 04 janvier 2021, consulté le 06 janvier 2021. URL : http://journals.openedition.org/formationemploi/7800 ; DOI : https://doi.org/10.4000/formationemploi.7800 


\section{La force de la forme. Discipline et socialisation des jeunes sans diplôme en internat d'inspiration militaire}

Jö̈L ZAFFRAN

Professeur de sociologie à l'université de Bordeaux. Membre du Centre Émile Durkheim (UMR

5116), et directeur du centre associé Céreq de Bordeaux

Résumé

La force de la forme. Discipline et socialisation des jeunes sans diplôme en internat d'inspiration militaire

L'Établissement pour l'Insertion dans l'Emploi (EPIDE) est un dispositif " Deuxième chance » dévolu aux jeunes sans diplôme. Son originalité est de les conduire vers l'emploi grâce à un accompagnement socio-éducatif en internat d'inspiration militaire. Pourquoi, alors que l'offre de raccrochage est variée, les jeunes sans diplôme ont-ils recours à un dispositif axé sur la rigueur et la discipline ? Notre enquête montre comment la nature instituante du dispositif s'ajuste à leur aspiration à l'autonomie et leur projet de vie conventionnel. Elle révèle que les jeunes s'engagent librement dans le dispositif, et comment la forme militaire améliore leur socialisation.

Mots clés : jeune, non diplômé, internat, socialisation, mesure jeune, méthode pédagogique, évaluation

Abstract

The strength of the form. Discipline and socialization of young people without diploma in military inspiration residency.

L'Établissement pour l'Insertion dans l'Emploi (EPIDE) is a "second chance" program for young people without a diploma. Its originality is to lead them to employment through a socio-educational program in a military inspirated internship. Why, while the offer of hang-up is varied, do young people without a diploma use an internship based on rigor and discipline? Our survey shows how the institutional nature of the internship adjusts to their desire for autonomy and their conventional life project. It reveals that young people engage freely in the internship, and how the military form improves their socialization.

Keyswords: young person, non graduate, boarding school, socialisation, youth employment scheme, teaching method, evaluation

Journal of Economic Literature: I 38 ; J 24

Traduction : auteur. 
Coincé dans la salle d'embarquement d'un aéroport de province, mon regard se pose sur une affiche entre deux panneaux publicitaires. Elle ne faisait pas la promotion d'un produit standardisé, mais vantait l'engagement dans l'armée. L'image m'est apparue d'abord énigmatique, moins par sa composition (la couleur verte dominante et le cadrage serré accentuent les symboles militaires) que la devise utilisée pour donner du sens à la structure d'ensemble. Le slogan était séduisant par ses allures d'oxymore : "J'ai rejoint les rangs pour sortir du lot" (cf. photo 1).

\section{Photo 1. Campagne de recrutement pour l'armée de terre. Source : Auteur}

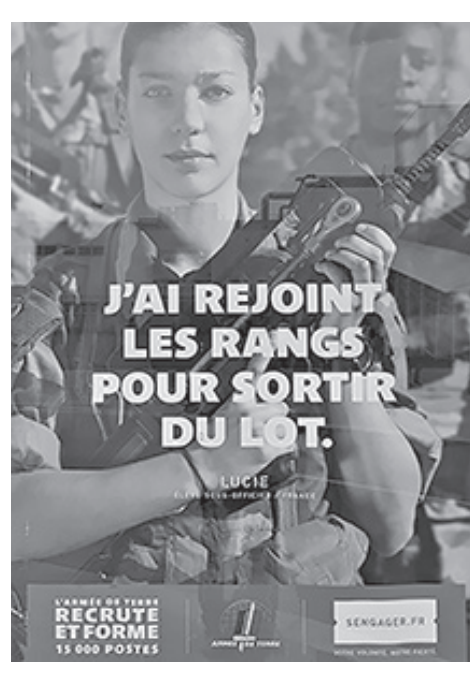

À y regarder de plus près, l'oxymore n'est pas dépourvu de sens. Le rapprochement de termes éloignés évoque deux attitudes consubstantielles du programme durkheimien d'éducation (la conformité sociale et l'individualisation) observées lors d'une enquête dans un dispositif d'inspiration militaire pour jeunes sans diplôme. Comment la discipline appuie-t-elle l'autonomie ? Quels sont les effets de la forme militaire sur les jeunes sans diplôme ? L'oxymore est le point de départ de cet article sur la (re)socialisation des jeunes sans diplôme qui font le choix d'un retour en formation en internat d'inspiration militaire ${ }^{1}$.

Un pan des politiques publiques de lutte contre le décrochage scolaire est consacré au retour en formation des jeunes sans diplôme. Des dispositifs " Deuxième chance " existent pour justement " raccrocher les décrocheurs ». Hormis les structures de retour à l'école de l'Éducation nationale (SRE), dont l'objectif est d'accompagner les raccrocheurs dans les épreuves du baccalauréat, le retour en formation consiste en un accompagnement des jeunes sans diplôme vers l'emploi.

Cet accompagnement peut se faire dans une École de la seconde chance (E2C) ou un Établissement pour l'Insertion dans l'Emploi (EPIDE). Dans le premier cas, l'alternance et l'individualisation sont au cour du dispositif'. Dès leur arrivée à l'E2C, les jeunes sont envoyés en stage en entreprise. Les apprentissages de base sont individualisés et transitent aussi par un outil informatique attestant que les compétences

\footnotetext{
1. Ce texte reprend la trame d'un article publié par Zaffran, 2015. Son originalité est d'en approfondir quelques thèmes, en particulier celui de l'autonomie des jeunes sans diplôme. L'auteur remercie le Conseil régional Nouvelle Aquitaine, ainsi que la Direction de l'évaluation, de la prospective et de la performance du ministère de l'Éducation nationale (tout particulièrement Caroline Simonis-Sueur) pour leur soutien.

2. Voir à ce sujet Zaffran \& Vollet (2018) ainsi que le dossier n ${ }^{\circ} 143$ de Formation Emploi. L'ouvrage et la revue proposent une analyse de la diversité des dispositifs de la "deuxième chance » et des parcours des jeunes sans diplôme qui retournent en formation dans ces dispositifs.
} 
sont acquises ${ }^{3}$. Dans le second cas, l'approche est très différente puisqu'elle fait appel à un accompagnement socio-éducatif des jeunes sans diplôme en internat d'inspiration militaire (voir encadré 1). Régi en partie par des articles du code de la Défense (Article L3414-1) et du code du service national (article L 130-1), l'EPIDE propose un accompagnement socio-éducatif assuré en grande partie par d'anciens militaires de carrière qui ont l'objectif de (re)socialiser les jeunes sans diplôme par l'emprunt ostensible à la codification militaire des règles de vie en internat.

\section{Encadré 1. Données de cadrage}

À sa création, I'EPIDE (Établissement pour l'insertion dans l'emploi) a été placé sous la tutelle des ministères chargés de la Défense, de la Ville et de l'Emploi. Il a été pensé comme une adaptation du service militaire adapté. Il est représenté par 18 centres, répartis sur 15 régions. Sur une année, il accueille environ 3400 jeunes (180 jeunes pour l'EPIDE de Marseille). Le taux de « sortie positive » (insertion professionnelle durable ou temporaire, retour en formation qualifiante) se situe autour de $50 \%$. Ce taux ne reflète pas le nombre de départs prématurés ( $40 \%$ en moyenne). D'ailleurs, la stabilisation des jeunes au sein de l'établissement est un but du contrat d'objectif et de performance signé en juin 2015. Le coût unitaire annuel par jeune est de 24000 euros environ. Le coût annuel à la place est de 49000 euros environ. Ces coûts sont élevés en raison des charges d'internat, et de l'importance du taux d'encadrement.

Tableau récapitulatif

\begin{tabular}{|l|c|}
\hline Nombre de jeunes (en flux annuel) & 3400 \\
\hline Taux de sortie en formation & $14 \%$ \\
\hline Taux de sortie en alternance & $7 \%$ \\
\hline Taux de sortie dans l'emploi durable & $17 \%$ \\
\hline Taux d'insertion dans l'emploi non durable & $10 \%$ \\
\hline Coût annuel tous jeunes confondus & $81 \mathrm{M€}$ \\
\hline Coût par jeunes (tous jeunes confondus) & $23900 €$ \\
\hline Coût annuel à la place & $49000 €$ \\
\hline Financement européen & $13 \%$ \\
\hline Financement État & $87 \%$ \\
\hline Financement Régions & $0 \%$ \\
\hline
\end{tabular}

3. L'attestation détaille les compétences acquises dans le domaine scolaire (par exemple, la maitrise des bases de la communication orale), technique (par exemple accueillir le client, identifier ses besoins et le conseiller) et socioprofessionnel (par exemple, être attentif à sa présentation, respecter les horaires demandés) acquises. 


\begin{tabular}{|l|c|}
\hline \multicolumn{1}{|c|}{ Origine de l'orientation locale } & $37 \%$ \\
\hline \multicolumn{1}{|c|}{ Journée Défense et Citoyenneté } & $7 \%$ \\
\hline \multicolumn{1}{|c|}{ Autres organismes } & $18 \%$ \\
\hline Candidature spontanée & $38 \%$ \\
\hline Délai d'entrée & en flux continus \\
\hline Rémunération & $\begin{array}{c}\text { entre 6 mois et 12 mois. Peut être prolongée sans excéder 24 } \\
\text { mois. La durée moyenne est de 7,5 mois }\end{array}$ \\
\hline Statut du jeune & $\begin{array}{c}\text { Allocation mensuelle et prime calculée au prorata du nombre } \\
\text { de mois de volontariat }\end{array}$ \\
\hline Objectifs & $\begin{array}{c}\text { Volontaire pour l'insertion (article } L \text { 130-1 du code du service } \\
\text { national }\end{array}$ \\
\hline
\end{tabular}

Sources : Cour des comptes, 2015, Les dispositifs et les crédits mobilisés en faveur des jeunes sortis sans qualification du système scolaire, Communication à la commission des finances, de l'économie générale et du contrôle budgétaire de l'Assemblée nationale et Cour des comptes, 2016, L'accès des jeunes à l'emploi. Construire des parcours, adapter les aides, Rapport public thématique.

\section{Implantation géographique des 19 centres EPIDE en 2019}

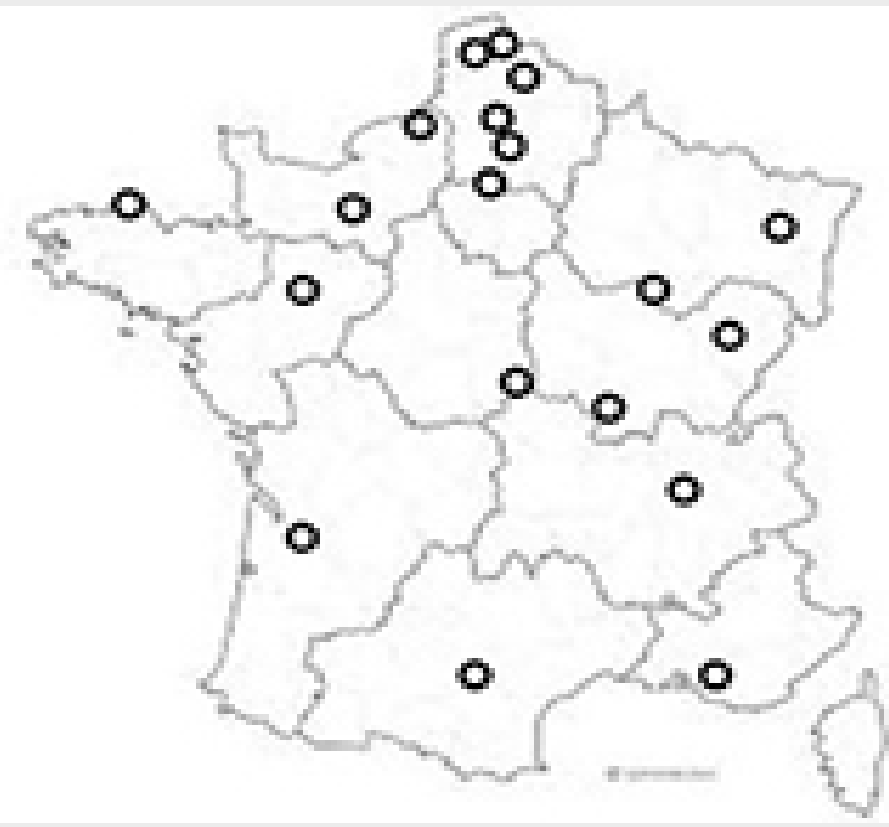

Alençon (61), Belfort (90), Bordeaux (33), Bourges-0smoy (18), Brétigny-sur-Orge (91), Cambrai (59), Combrée (49), Doullens (80), Langres (52), Lanrodec (22), Lyon-Meyzieu (69), Margny-lès-Compiègne (60), Marseille (13), Montry (77), Saint-Quentin (02), Strasbourg (67), Toulouse (31), Val-de-Reuil (27), Velet (71). Source : EPIDE, https://www.epide.rr/a-propos/les-centres-de-lepide. 
Le socle militaire de l'accompagnement confère à l'EPIDE une originalité qui rappelle le programme Job Corps mis en place aux États-Unis dans le contexte de "guerre contre la pauvreté " menée dans les années $1960^{4}$. Ce programme "d'éducation résidentielle " accueille en internat des jeunes en difficultés et les accompagne vers une insertion professionnelle par l'apprentissage de compétences sociales et des savoirs de base nécessaires à leur " employabilité ". Dans la tradition américaine de l'évaluation, utilisée comme la démonstration de l'utilité de l'action publique et le moyen de légitimer des politiques sociales controversées (Lacouette Fougère \& Lascoumes, 2013), ce programme a fait l'objet d'un rapport qui souligne les effets économiques sur l'emploi et la délinquance, et pointe aussi les effets sur l'estime et la confiance en soi des jeunes eux-mêmes (Schochet \& al., 2008).

L'EPIDE ressemble, à bien des égards, à Job Corps : l'accompagnement se fait en internat, les règles y sont strictes, et les jeunes sont volontaires. Préalablement à leur arrivée, ils ont assisté à une séance d'information et passé un entretien de motivation. Ils doivent aussi signer un " contrat de volontariat pour l'insertion » qui rappelle leurs droits, mais aussi leurs obligations : participer activement à la formation, suivre assidument toutes les activités, respecter scrupuleusement le règlement. Ainsi, le dispositif est bien décrit lors des séances de pré-admission, de façon plus minutieuse encore lors de l'entretien d'entrée. De plus, les jeunes gardent la possibilité de ne pas signer le "contrat de volontariat pour l'insertion " avant leur admission" 5 . De ce fait, la discipline et la connotation militaire de l'accompagnement ne les surprennent pas. Dans ce cas, pourquoi ont-ils recours à ce dispositif ?

La question ne peut pas être traitée sans d'abord déconstruire l'image des décrocheurs scolaires qui présume des réticences à adhérer aux normes sociales et scolaires, mais aussi les assimile à une population en incapacité de partager le destin commun de leur génération (Maillard \& Rouaud, 2018). Elle doit ensuite articuler la finalité objective du dispositif d'insertion des jeunes sans diplôme avec les effets subjectifs de la formation sur cette autre jeunesse qui désigne les jeunes démunis de ressources hormis une ressource corporelle (Dubar, 1987). Se préparant à des emplois non qualifiés qui réclament, la plupart du temps, de la force physique, les jeunes élaborent des stratégies plus ou moins complexes d'intégration sociale qui envisagent la socialisation comme un préalable à l'insertion professionnelle. En d'autres termes, le retour en formation des

\footnotetext{
4. Job Corps est un programme fédéral de formation professionnelle des jeunes défavorisés. Il fournit des services d'éducation de base, de compétences professionnelles, de soins de santé, de conseils et d'hébergement. L'objectif est d'aider les jeunes à "devenir des citoyens plus productifs et plus employables " (cf. Burghardt J., Schochet P., 2011, National Job Corps Study: Impacts by Center Characteristics, evaluative report, Employment and Training Administration (DOL), Washington, DC. Office of Policy and Research).

5. Nous avons assisté, dans une mission locale, à une séance de présentation et d'information de l'EPIDE par une formatrice. Les échanges avec les jeunes furent directs, et les réponses toujours très précises. La formatrice n'a eu de cesse de rappeler les contraintes du dispositif, sa discipline et ses règles.
} 
jeunes sans diplôme à l'EPIDE est une stratégie d'insertion professionnelle indexée sur leur (re)socialisation par la réorganisation de l'espace et du temps. Ce faisant, notre définition de la jeunesse et de la socialisation va dans le sens d'une sociologie de l'expérience (Dubet, 2009 en particulier le chapitre 3 et Dubet, 2018) qui porte sur le travail des personnes pour gérer la tension entre leur émancipation personnelle, leurs investissements pour acquérir le statut d'adulte et les contraintes, économiques ou sociales, pesant sur le parcours individuel. Pour notre part, on reconsidère le parcours à l'aune des normes collectives issues du dispositif d'inspiration militaire (Zaffran, 2015). En conséquence, notre hypothèse est que la forme militaire $e^{6}$ est choisie pour sa fonction de transition objective vers l'emploi et un statut social, mais aussi pour les transactions physiques et subjectives avec soi-même et avec autrui. L'EPIDE est assimilable à une quête des honneurs qui vise une réhabilitation sociale et symbolique et qui réclame des efforts sur soi pour être menée convenablement. L'article propose de le démontrer par l'interprétation des éléments issus d'une observation directe dans un EPIDE7 .

Dans une première partie, il présente les principes généraux du dispositif, avec une insistance particulière sur le contenu de la forme militaire. Dans une deuxième partie, il montre comment la forme militaire socialise les jeunes, et prépare leur transition biographique. Sans occulter les limites de l'enquête, la conclusion est l'occasion d'interroger les vertus et les écueils du caractère instituant du dispositif ${ }^{8}$.

\section{Encadré 2. Méthodologie}

Les données proviennent d'une monographie d'un EPIDE (Établissement pour l'insertion dans l'emploi) situé dans une zone urbaine sensible d'une métropole du sud de la France (voir encadré 1). Cerné par l'autoroute et des blocs d'HLM (habitation à loyer modéré) dégradées, l'établissement respecte le plan architectural d'une caserne au sens premier du terme, c'est-àdire un endroit destiné à loger les troupes, plus largement de lieu où règne un « style de vie ». L'entrée se fait par un portail principal qui ne s'ouvre qu'après avoir décliné son identité. La barrière levée, on pénètre à l'intérieur d'une enceinte protégée par des murs. On est pris immédiatement par le sentiment d'être coupé d'un environnement que l'état dégradé de la route, l'abandon des carcasses de voitures calcinées et la vétusté des immeubles rendent inhospitalier (cf. photo 2).

6. La forme militaire désigne un accompagnement socio-éducatif fondé sur la discipline militaire. Elle est une configuration dynamique de composantes martiales dont l'interrelation crée les conditions d'appropriation d'une discipline qui organise les activités scolaires et physiques. La notion est définie avec précision dans la deuxième partie de l'article.

7. Voir encart méthodologique.

8. On entend par dispositif l'organisation sociale et pédagogique au service d'un accompagnement social et d'une insertion professionnelle des jeunes sans diplôme pris en charge. 


\section{Photo 2. Vue aérienne de l'EPIDE. Source : Google}

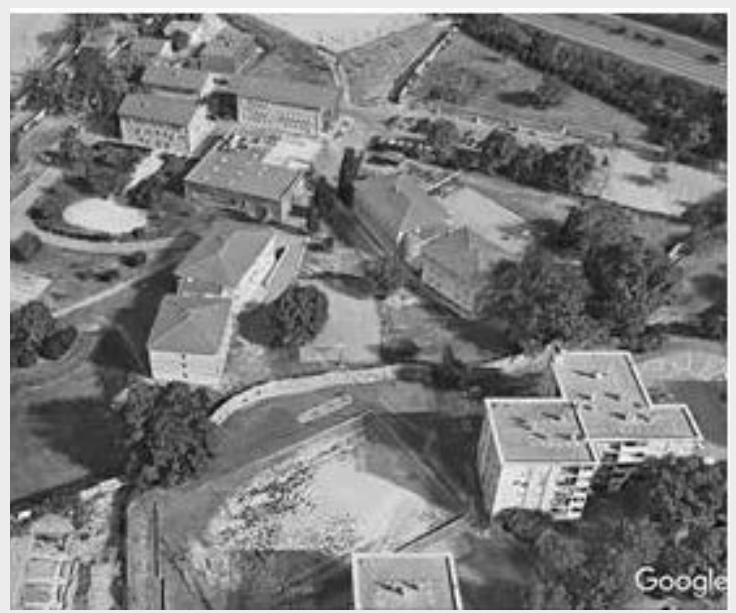

Les bâtiments sont répartis entre une zone administrative, une zone d'activités scolaires et sportives, une zone de vie et d'hébergement; enfin une sorte de place d'armes où se déroulent les rassemblements collectifs. De larges travées facilitent la circulation des groupes de jeunes se déplaçant en rangs ordonnés. L'ordre et la solennité sont palpables à travers les symboles et les rituels militaires.

Notre séjour de plusieurs semaines dans cet EPIDE était consacré à l'étude du fonctionnement du dispositif et de son organisation. Outre des observations directes, nous avons réalisé des entretiens avec les jeunes (une trentaine d'entretiens, répartis à parts plus ou moins égales entre des filles et des garçons, dont certains - une dizaine - sont originaires d'un territoire ultramarin, notamment de Mayotte).

Ces jeunes, âgés entre dix-huit et vingt-trois ans sont pour la plupart issus d'un milieu infrapopulaire et résident dans un quartier sensible. Ils ont un parcours scolaire identique et vivent dans des conditions sociales assez proches. Ils sont représentatifs de l'ensemble des jeunes du dispositif. Sur un plan sociodémographique, cet EPIDE est composé, au moment de l'enquête, à plus de $70 \%$ de garçons. La moyenne d'âge est de 19,5 (écarts-types 2 ans). Leur nationalité est française pour l'immense majorité (18\% environ nés à Mayotte, et $4 \%$ aux Comores). Sur le plan familial, $81 \%$ déclarent vivre avec leur famille, $3 \%$ en couple et $16 \%$ seul-es. Par ailleurs, $62 \%$ signalent que leurs parents sont séparés. Pour plus de $60 \%$, le père est sans emploi. Les parents qui travaillent occupent pour la plupart des emplois d'employés ou d'ouvriers. Parmi les jeunes, $28 \%$ ont déjà été en garde en vue, $15 \%$ ont eu une peine avec sursis et $6 \%$ ont connu la prison. Sur le plan scolaire, ils sont $74 \%$ à avoir redoublé une ou deux fois durant leur scolarité. L'arrêt des études est intervenu au collège ou en lycée d'enseignement professionnel ( $29 \%$ dans le premier cas, $64 \%$ dans le deuxième cas), pour une faible part en apprentissage (7\%).

$\left.{ }^{*}\right)$ : La plupart des EPIDE sont des casernes militaires désaffectées ayant fait l'objet d'une réhabilitation à la suite une cession d'emprise immobilière par le ministère de la Défense. Certains EPIDE, bien qu'implantés dans des bâtiments neufs, respectent l'architecture des casernes. Source : enquête par questionnaires, données extraites selon la ville concernée. 


\section{Les perspectives temporelles du recours au dispositif}

Lancé en 2005 par la voix du Premier ministre, qui se déclare "favorable à la transposition sur le territoire métropolitain du savoir-faire des armées en matière d'insertion professionnelle, dont l'efficience avait été prouvée dans le cadre du service militaire adapté ", la finalité de I'EPIDE est de "mettre les compétences des anciens militaires au service d'un projet de société tournée vers la jeunesse en difficulté d'insertion professionnelle »".

Il se distingue des autres dispositifs " Deuxième chance " par les trois principes de son accompagnement socio-éducatif : 1- un (ré)apprentissage des règles de vie en société (également appelé formation comportementale) ; 2- un rappel des principaux acquis scolaires (voire, pour certains jeunes, une remise à niveau) ; 3 - une formation "préprofessionnelle " s'appuyant à la fois sur l'expérience d'insertion des armées et sur les dispositifs d'insertion existants. La direction de l'EPIDE est assurée par un officier de l'armée de l'air à la retraite, qui a la responsabilité des pôles administratif, technique, pédagogique et d'encadrement. Le pôle pédagogique et le pôle encadrement se composent de cadres, de formateurs et de moniteurs. Le personnel est composé d'anciens militaires du rang et de formateurs issus du champ de l'animation, de l'éducation populaire ou de l'intervention sociale. Les formateurs sont chargés de l'éducation civique et scolaire. Des chargés d'insertion professionnelle s'occupent des stages, tandis que les moniteurs veillent au respect des règles de vie en collectivité.

La tenue réglementaire, en l'occurrence une parka, ainsi qu'un pantalon bleu marine et des chaussures noires ( $c f$. photos 3 et photo 4 ), est portée par les jeunes et les adultes. La journée démarre à 6 heures du matin et se termine à 23 heures. Elle est occupée par des activités scolaires, des ateliers et des accompagnements professionnels auxquels ils se rendent par petits groupes, en rang et au pas. Les garçons sont rasés de frais, tandis que les filles sont peu maquillées et ont les cheveux suffisamment tirés pour ne pas toucher le col de la chemise. Le vouvoiement est de rigueur entre les adultes et les jeunes. L'accompagnement des jeunes se conforme au règlement assez strict qui organise le temps et l'espace.

Les premières semaines dans le dispositif sont consacrées à l'apprentissage des postures militaires du corps. Outre la marche au pas, répétée jusqu’à la régularité, les jeunes apprennent à rester immobile, et à se tenir droit (cf. photo 3$)$.

9. Il s’agit du discours de politique générale prononcé par le Premier ministre, Dominique de Villepin, le 3 juin 2005. Le lecteur peut aussi se référer au rapport d'information du Sénat n 290 (2007-2008) déposé au nom de la Commission des finances le 16 avril 2008 (Disponible : https://www.senat.fr/rap/r07-290/ r07-290.html) 
Photo 3. Consignes au garde-à-vous. Source : AFP

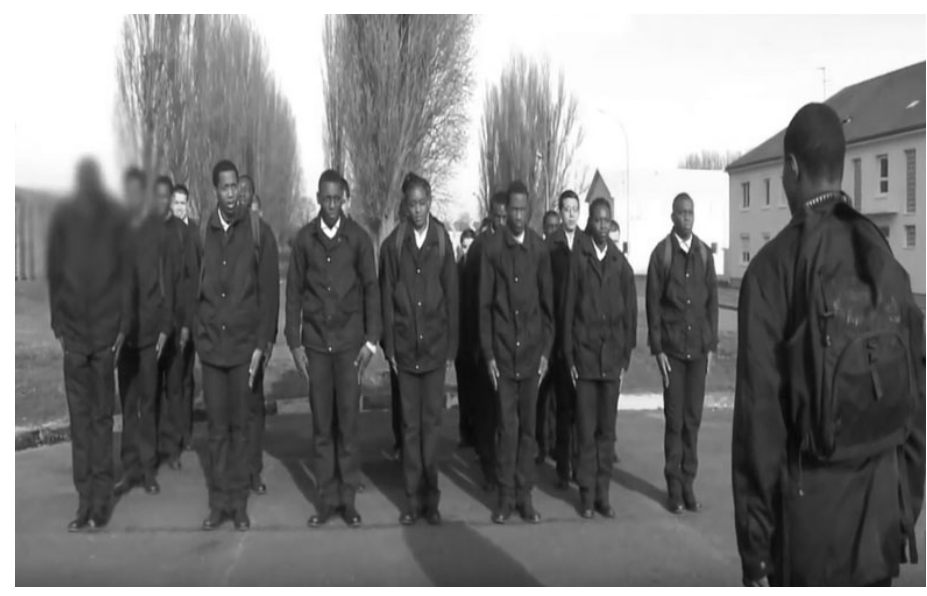

Chaque vendredi matin, à 8 heures précises, les adultes et les jeunes se rassemblent pour la cérémonie du drapeau sur ce qui s'apparente à une place d'armes. Deux jeunes, en général choisis parmi les plus méritants de la semaine, hissent le drapeau tricolore devant toute l'assemblée au garde-à-vous (cf. photo 4) qui entonne l'hymne national. Le directeur fait ensuite un résumé de la semaine écoulée, puis revient sur les fondements de la vie en internat et de la discipline. Ce moment, qui succède à la cérémonie du drapeau, s'apparente à une cérémonie de dégradation et de récompense en public, lors de laquelle les réprimandes faites aux uns par le directeur interviennent toujours avant les félicitations et les gratifications symboliques adressées aux autres.

Photo 4. Rassemblement hebdomadaire à I'EPIDE. Source : auteur

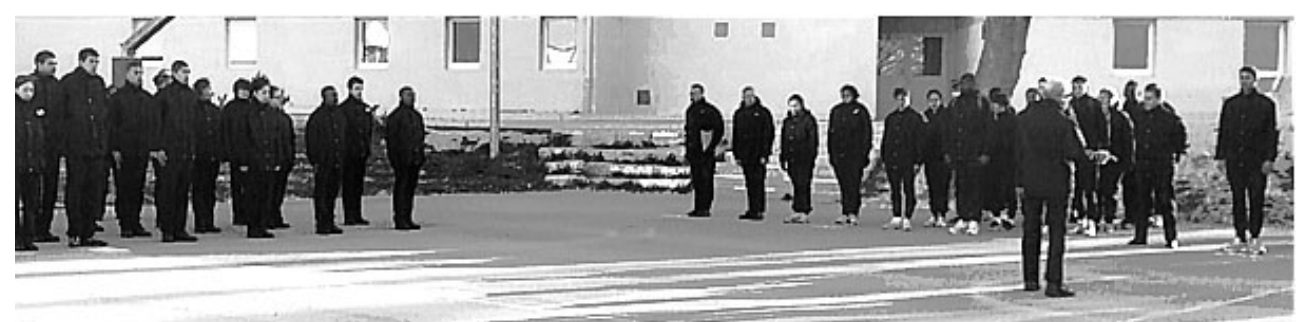

L'EPIDE s'adressant à des jeunes éligibles aux aides financières à la formation professionnelle et l'accompagnement faisant appel aux attributs ostensibles de l'étiquette militaire, les motifs du recours des jeunes à ce dispositif seraient, de prime abord, de deux ordres.

Le premier serait le motif économique. À leur arrivée, les jeunes perçoivent, au titre du statut de stagiaire de la formation professionnelle, une allocation mensuelle d'un montant de 300 euros environ dont une part est capitalisée sur un compte bancaire à leur nom, et reversée en fin de parcours. On pourrait penser que ce pécule est à l'origine de leur engagement. Or, moins de la moitié des jeunes interviewés déclarent apprécier la rétri- 
bution financière ${ }^{10}$. Ceux et celles ayant mentionné le versement d'une allocation comme un motif du recours à l'EPIDE s'empressent de dire qu'elle n'est pas déterminante dans leur choix : "Franchement, avec mon bizness" ${ }^{11}$, je gagne beaucoup plus dehors, c'est pas ça qui motive " (Bilal, 18 ans, vit avec sa mère agent de surface, ne sait pas où vit son père, a arrêté en $1^{\text {er }}$ année de CAP Serrurerie); "C'est sûr que les sous quills mettent sur le compte, ça peut aider, mais sérieux, tu vas pas loin avec" (Sofia, 19 ans, vit avec ses parents, son père est ouvrier et sa mère est femme au foyer, a arrêté en $2^{\mathrm{e}}$ année de CAP Hôtellerie). Ainsi, l'allocation versée n'explique que partiellement l'engagement dans le dispositif.

Le second motif serait lié à l'image de l'armée dans une société marquée par l'affaiblissement de l'antimilitarisme. Le déclin des représentations négatives associées à l'armée (Léger, 2005 ; Hatto \& al., 2011) pousserait plus facilement les jeunes sans diplôme à y faire carrière : nombre de jeunes rencontrés ont insisté sur les possibilités offertes par l'armée, aux non diplômés, d'une insertion professionnelle et d'une véritable carrière. L'EPIDE serait l'antichambre de l'armée parce qu'il prépare aux épreuves sportives et psychotechniques du recrutement des engagés volontaires de l'armée. Si certains jeunes (qu'il s'agisse des garçons ou des filles) visent la gendarmerie, la police ou l'armée, d'autres ont des projets éloignés des forces armées ou de la protection civile. À y regarder de près, la carrière militaire n'intéresse que la moitié des jeunes ${ }^{12}$, en particulier les plus démunis sur le plan scolaire (ils ont arrêté très tôt leur scolarité) et ceux originaires d'un territoire ultramarin. Ceux-là se livrent corps et âme dans la formation, et par conséquent se plient aisément aux contraintes. La proximité avec d'anciens militaires est à leurs yeux une ressource importante pour connaître les métiers de la Défense, et être préparés convenablement aux épreuves d'admission ( $c f$. infra le cas de Brandon). Ce rapport au dispositif est aussi celui des jeunes dont le parcours scolaire plus avancé hisse leurs aspirations vers un métier dans la protection civile et le secours aux personnes. Pour eux, l'EPIDE est un moyen de préparer les épreuves physiques, orales et écrites du concours d'adjoint de sécurité, qu'ils voient comme un préalable au métier de gardien de la paix, de gendarme ou de pompier.

Alors que la formation va de pair avec une allocation, les jeunes ne font pas de l'argent un motif principal du recours à l'EPIDE, et alors que l'esprit militaire du dispositif aurait pour fonction une socialisation professionnelle anticipatrice dans la Défense, tous n'ont pas un projet professionnel en lien avec l'armée. Il faut donc admettre que le pécule et le projet professionnel dans l'armée ne suffisent pas à comprendre le phénomène ${ }^{13}$, et par conséquent à trouver un motif commun à tous les jeunes de recourir au dispositif. En s'ins-

10. Dans le questionnaire, ils ne sont que $38 \%$ à déclarer avoir décidé d'entrer à l'EPIDE pour des raisons financières.

11. Bilal revendait du cannabis.

12. $50 \%$ environ citent un métier dans la Défense comme projet professionnel.

13. Le questionnaire montre que $63 \%$ ont choisi l'EPIDE pour la discipline, quand la moyenne à l'échelle de satisfaction de l'accompagnement des formateurs (de 0 pas du tout à 10 totalement) est de 8.4 (et 2.44 d'écart-type). 
pirant de la théorie de l'éligibilité, on suppose que le recours s'analyse comme une demande séparée du futur et du présent (Lévy-Garboua, 1976). Le futur renvoie au marché du travail auquel les jeunes espèrent accéder. Quand on interroge les jeunes sur les raisons de l'acceptation des contraintes du dispositif, les réponses ont moins des allures d'une résignation que d'un arbitrage entre ce que l'EPIDE oblige à faire et ce qu'il peut offrir : l'espoir d'une insertion professionnelle rapide en échange d'une observance du règlement et du respect de la discipline. Les règles ne sont pas ressenties comme une contrainte dès lors qu'elles apparaissent comme une condition de l'accès au marché du travail. En l'espèce, les jeunes rencontrés adhèrent tous à la rhétorique des formateurs selon laquelle la forme militaire, qu'il faut de toutes façons subir, est un atout face à des employeurs attentifs aux prérequis comportementaux indispensables pour obtenir puis tenir un emploi : ponctualité, régularité, application. Le présent renvoie aux biens non marchands que produit immédiatement le dispositif de formation, ce qui conduit à considérer que les substrats matériels, techniques et organisationnels - de la forme militaire produisent à l'EPIDE des effets similaires aux écoles militaires. En premier lieu, les cérémonies collectives, les rituels et les symboles militaires fabriquent de la cohésion et de la solidarité (Léon, 1999). En deuxième lieu, ces valeurs participent à l'expérience des jeunes sans diplôme, à l'instar des jeunes diplômés formés dans une grande école sous statut militaire qui déclarent y vivre " une expérience fortifiante ${ }^{14}$ » (Weber, 2012 ; Allouche, 2017).

\section{La force 2 de la forme}

Ainsi, la forme militaire est à la fois le ressort du recours et le moteur de l'engagement des jeunes dans le dispositif par les effets subjectifs attendus et produits. Sur un plan conceptuel, la forme militaire évoque ; d'une part, le concept de forme que Vincent (1980) applique aux dispositifs scolaires, d'autre part, la militarisation des relations sociales. Dans les dispositifs scolaires, la forme scolaire désigne des savoirs supposés stables que des formés acquièrent dans un rapport pédagogique au formateur, ce dernier incarnant une autorité juste et neutre. Il punit et récompense sans jugement de valeur, sur la simple règle de l'erreur et du succès. Il est guidé par un principe de dépersonnalisation de sa relation aux élèves ${ }^{15}$.

La discipline au principe de ce rapport assure le consentement des formés par le contrôle scolaro-centré des procédures réglementaires dans le temps et dans l'espace. Comme le

14. Le sentiment d'appartenance à une communauté est si fort que certains jeunes se désignent d'eux-mêmes comme des " épidiens ». En cela, le dispositif fortifie le travail de subjectivation par la logique d'intégration. 15. Malgré les inflexions du système éducatif, et bien que les relations des enseignants aux élèves évoluent, la forme scolaire perdure. 
note Barrère (2013, p. 97), c'est le double sens du mot discipline, à la fois une contrainte par des procédures scolaires objectives et la partition curriculaire d'un ensemble de savoirs.

En cela, la forme scolaire a des points communs avec la forme militaire. L'une et l'autre répartissent les jeunes dans des groupes, et dispensent un enseignement organisé autour de savoirs spécifiques. Dans les deux cas, leur progression se fait à partir d'un découpage précis des compétences, ainsi qu'une formalisation du contenu des connaissances scolaires de base. De plus, elles reposent sur des relations impersonnelles d'autorité exercée dans un contexte de séparation spatiale et de réorganisation temporelle (Pirone, 2015).

La forme scolaire et la forme militaire se rejoignent donc sur les modes de socialisation par une organisation précise du temps et de l'espace, mais elles se différencient par la nature des expériences. D'abord, l'objectif de " renormaliser les comportements » des jeunes est assumé par l'EPIDE, quand l'objectif de renormalisation des élèves "difficiles " de l'école passe par un infléchissement de la forme scolaire à laquelle il faudrait les réapprivoiser (Pirone \& Rayou, 2012). Ensuite, et plus que dans les dispositifs de retour à l'école, l'expérience est de nature "fortifiante ", comme on l'a vu. La «socialisation épidienne " agit sur la personnalité dès les premiers jours, lors de la "semaine d'intégration ». Pendant cinq jours, les jeunes passent des épreuves physiques à l'extérieur de l'établissement, avec des objectifs de performance qui servent à classer le groupe plutôt que le jeune : « La semaine d'intégration, c'est trop bien. Wallah, c'est dur, y en a plein qui craquent. Mais à la fin, on est tous contents. C'était un super moment. On s'aidait, on sencourageait et tout. Maintenant, je sais que je peux compter sur les autres si je craque. Je sais aussi que mes formateurs ne me traitent pas comme les profs miont traité" (Abdullah, 20 ans, vit avec ses parents, sa mère fait des ménages et son père est au chômage, a arrêté en $2^{\mathrm{e}}$ année de BEP électricité). Les épreuves physiques font appel autant à l'affirmation de soi qu’à la solidarité. Elles réparent aussi une estime de soi malmenée notamment par l'expérience scolaire. Ces valeurs expliquent l'attachement revendiqué par les jeunes aux cérémonies militaires, et que Keck (2012) aide à comprendre : elles les hissent au niveau d'une morale sociale par leur participation à des actions collectives ; ce faisant, elles leur ouvrent la voie à des émotions collectives ainsi qu'au respect de soi et des autres. Au même titre que les épreuves physiques lors de la semaine d'intégration, les rituels militaires sont les aiguillons faisant ressentir la force du groupe, et à travers lui, le fait de se sentir soi. Autrement dit, ils font l'interface entre les règles objectives du dispositif et la subjectivation. Enfin, les règles autonomes et rationnelles, autrement dit dépersonnalisées, qui organisent la vie quotidienne font ressentir le dispositif comme un lieu « hors du monde » qui protège des désordres du quartier. La culture de rue n'est pas prédominante dans la mesure où le règlement ne la tolère pas, et que l'enceinte coupe le dispositif de l'environnement : "Je me sens en sécurité ici, franchement, parce que dans mon quartier, ça craint trop, des fois, même en plein ramadan, ça tire le soir, les darons (les parents) ils cavalent pour rentrer chez eux, ça crie casse-toi, dégage de là et tout. Ici, c'est mieux que dehors, on est plus cool, je sais pas, comme si on était à l'abri” (Hamid, 19 ans, vit chez ses parents, la mère est femme au foyer et le père magasinier, a arrêté en $2^{\mathrm{e}}$ année de CAP carrosserie, n’a pas de projet professionnel). 


\subsection{Grandir et s'émanciper}

Au fond, le dispositif est un travail socio-éducatif axé sur la discipline et la militarisation de l'accompagnement que les jeunes endurent d'autant plus facilement qu'ils sont les auteurs de leur histoire. Le dispositif donne du sens à l'engagement des jeunes en les polarisant sur des objectifs qu'ils ont élaborés avant d'entrer à l'EPIDE.

Et même si les filles et les garçons n'entrent pas toutes et tous à l'EPIDE avec un projet professionnel précis, les unes et les autres le voient comme une transition biographique par les rôles dans lesquels il les installe et les prépare le temps de la formation : celui de "frère d'armes " ${ }^{16}$ lors des épreuves physiques, d'élève au moment des séquences scolaires, de travailleur lors des stages de préprofessionnalisation. Plus largement, ils le ressentent comme une transaction, avec soi et avec autrui, grâce à laquelle évolue l'idée qu'ils ont d'eux-mêmes.

En somme, ils grandissent : "tous ces trucs, ça prend la tête, mais quand tu les fais, c'est comme si tu deviens un homme" dit Assedine sur un ton emphatique, quand Luna déclare plus sobrement qu' «à l'EPIDE, on devient adulte». Il est remarquable que les jeunes évoquent souvent la crainte du retard pris sur les agendas sociaux, mais l'expriment différemment. Les jeunes originaires des territoires ultramarins français (en particulier de Mayotte), principalement des garçons, assimilent l'EPIDE à une préparation militaire, mais le voient aussi comme un rite de passage à l'âge adulte par les souffrances endurées. La forme militaire est vécue comme une mise à l'épreuve de soi qu'ils surmontent avec la conviction, comme l'affirme Ali en se tapant la poitrine avec le poing, qu' être militaire, c'est être un homme ». Ils relatent longuement les exercices physiques qui demandent résistance et courage, auxquels ils se soumettent sans renâcler, en «serrant les dents " pour ainsi dire : «Faut rien lâcher. Même si j’ai mal aux jambes et que c'est la mort, je lâche rien " (Hussein, 19 ans, ses parents vivent à Mayotte).

Devenir un homme est l'objectif conforme aux représentations populaires et culturelles de l'idéal de virilité. Il faut donc faire ses preuves face aux pairs, plus largement aux aînés de son entourage. Les épreuves sont des moments forts dans leur expérience car elles annulent, au moins temporairement, les traits personnels et sociaux qui définissent les jeunes à l'extérieur. Plus la volonté de rompre avec le passé est forte et plus l'adhésion aux connotations viriles des épreuves est robuste, plus la loyauté envers le dispositif est puissante. Désormais, le présent est tout entier soumis au regard des autres.

C'est pourquoi une partie des tensions entre jeunes provient précisément des cas d'indiscipline, et que le comportement déviant de certains est sévèrement jugé par le groupe, puisque l'atteinte à l'autorité du cadre est vécue comme une atteinte à l'identité sociale à laquelle prépare le dispositif : "Y en a ils font le bordel le soir dans les chambres ou qui se font remarquer la journée. Je comprends pas. Avec les autres, on se dit, pourquoi ils sont là? Si

16. Pour reprendre l'expression qu'un jeune emprunte au langage militaire. 
tu viens à l'EPIDE, c'est pour toi. Tu l'as choisi, non? C'est pour changer, devenir quelqu'un. Si c'est pour pas changer, c'est pas la peine, faut rester au quartier. Moi, je sais ce que je veux, je suis déterminé» (Kevin, 20 ans, vit chez ses parents, sa mère fait des ménages et son père est sans emploi, a le projet d'entrer dans la sécurité).

Les ressorts de l'adhésion au dispositif ne sont pas univoques, car des différences existent entre garçons et filles. On présuppose qu'elles sont dues à la socialisation au sein de la famille et au sein du quartier ou de l'école. Les modèles familiaux qui fondent l'apprentissage des rôles selon le sexe donnent lieu soit au renforcement des normes culturelles, soit à une déconstruction des identités sexuées.

Il reste que les entretiens avec les premiers évoquent la vision traditionnelle du rôle masculin qui préside à leur accès à l'EPIDE. Le désir de fonder rapidement une famille et de disposer d'un logement à soi revient souvent dans les entretiens. Les jeunes issus d'un territoire ultramarin se différencient par leur insistance à tenir leurs rôles masculins, d'autant plus qu'ils considèrent que l'âge de se marier est venu, et que le poids des normes culturelles rend pressant le besoin de revenus, c'est-à-dire d'indépendance financière. Ils s'accrochent à l'EPIDE dans l'espoir de "réussir ", c'est-à-dire d'être reconnus comme des adultes par la communauté avec laquelle ils n'ont jamais rompu les liens, et qui les regardait, sinon comme des enfants, du moins comme des individus en liminalité. La forme militaire engage le processus de validation du statut de membre de la communauté que l'insertion professionnelle parachèvera.

Cette vision traditionnelle du rôle masculin contraste avec celle que les filles ont du rôle féminin. Toutes issues d'un quartier difficile, elles endurent la forme militaire moins par adhésion aux valeurs militaires de virilité ${ }^{17}$ que par opposition à la place des femmes dans la famille, le quartier ou la communauté : "Les garçons, ils croient quion va leur faire la cuisine, le ménage et tout ça. Ils peuvent courir. Moi, je suis ici pour me tailler de chez moi." (Sarah, 19 ans, vit avec ses parents, sa mère fait des ménages et son père est maçon, a arrêté en 2e année de BEP sanitaire et social). Leur engagement prend alors des allures de défi vis-à-vis des rôles traditionnels. Si elles éprouvent plus de difficultés à entrer à l'EPIDE (la décision est souvent prise contre l'avis des parents ou de la famille), une fois le sésame en main, elles retournent à leur avantage les épreuves de l'EPIDE, qui deviennent les supports de leur émancipation. Dès lors qu'elles en sortent victorieuses, elles prouvent aux garçons (de l'EPIDE et du quartier) qu'elles sont capables de les endurer de manière identique. De plus, l'enceinte sépare d'un contexte familial qu'elles sont plusieurs à décrire comme pesant, voire plus contraignant que la forme militaire elle-même. La surveillance étroite qu'elles subissent à l'extérieur, redoublée parfois d'un confinement dans l'espace domestique (par opposition à la culture de rue des garçons), oblige à une double stratégie d'indépendance financière par le retour en formation comme préalable à l'insertion pro-

17. Nous avons observé que certaines filles adoptaient, voire surjouaient ces valeurs de virilité, comme si l'emphase facilitait leur entrée dans un monde qu'elles pensent réservé aux hommes. 
fessionnelle, et d'émancipation par la mise en scène de soi dans une arène virile. Cette arène n'est pas conçue comme une ressource (autrement dit comme une information sur le dispositif aux cadets, qu'elles pourront redistribuer à leurs frères (Beaud, 2018)), mais comme le moyen de maîtriser leur propre situation et plus largement leur destin. Elles accumulent les ressources du dispositif pour leur propre compte, et dans le même temps veulent peser par elle-même sur leur trajectoire en faisant leurs preuves devant les garçons et devant leur entourage.

Au final, les garçons voient le dispositif comme un univers viril qui confirme la disposition au rôle conventionnel et aux représentations intériorisées dans les milieux populaires de monsieur gagne-pain, tandis que les filles se glissent dans la forme militaire pour mieux se démarquer du rôle, trop traditionnel à leur goût, de madame au foyer.

\subsection{Une autonomie variable}

Si la forme militaire n'est pas rejetée par les jeunes, c'est qu'ils y trouvent un programme au sens institutionnel du terme, c'est-à-dire un modèle de socialisation dérivée d'une forme rendue stable par le socle militaire. L'accompagnement s'arrime aux principes de justice que l'armée incarne, de sorte que les normes de conduite et les règles de vie imprègnent plus la personnalité que les contenus transmis.

D'ailleurs, la stabilité des principes, la forme, donne lieu à une expérience épidienne qui contraste avec les expériences scolaires : "Ici, c'est pas comme à l'école. Tu te défonces, on te récompense. Franchement, j'ai de la valeur ici, je suis quelqu'un parce que quand je fais les choses, on me dit c'est bien, continue, on me félicite et je sais que c'est pour mon bien. À l'école, je travaillais comme un malade, la vérité, et j'avais rien en retour. Les profs, ils me disaient tu travailles pas. J'avais trop la haine. " (Akim, 18 ans, vit avec sa mère employée, a arrêté en CAP Plomberie)

Alors que l'école était vécue comme une machine à trier ou, pire encore, un appareil de distribution sur des critères ethniques ou sociaux ("Dans ma classe, c'était que des rebeus et des renois " ${ }^{18}$ ajoute Akim), l'EPIDE est vu comme une " fabrique d'universel " par la réussite de tous qui dépend de la volonté de chacun. L'égalité, qui s'incarne dans l'uniforme et les valeurs militaires, précède le mérite auquel les jeunes veulent croire car il individualise leur engagement en les rendant dépositaires de leur réussite devant leurs pairs. En cela, le dispositif est une évocation du programme institutionnel qui éduque et autonomise par une socialisation dont les effets symboliques et collectifs proviennent de la soumission à une discipline rationnelle et collective (Dubet, 2010).

La forme militaire de l'EPIDE est libératrice précisément parce qu'elle repose sur la reconnaissance du mérite. C'est d'ailleurs ce qui rend la discipline acceptable puisqu'elle est une condition de la réussite, mais aussi de l'intégration dans le dispositif. La discipline " corrige » le corps et oblige à régler sa conduite sur celle des autres. En retour, le groupe accom-

18. Arabes et noirs en verlan. 
pagne le combat contre ses propres " désordres ", dans un corps-à-corps avec soi-même, mais sous le regard de tous. L'autonomie est donc le départ et le résultat d'un programme somme toute durkheimien. Ce programme ne conçoit pas la faculté de se gouverner soi sans la soumission volontaire à des règles collectives, et reconnaît le talent des engagés dans les épreuves proposées ; par conséquent, il justifie le différentiel d'attribution de jugement et de valeur moins sur des critères scolaires et ethniques que personnels.

Bien que coupé de l'extérieur, l'internat a des effets sur les jeunes dans la limite des entraves et des projets construits avant de débuter la formation, et que le dispositif ne peut pas lever. Dès lors, l'autonomie se décline de trois manières. Elle est d'abord le résultat d'un investissement dans la formation qui gagne en intensité quand une recherche active d'emploi existe avant de débuter la formation. La solidité de leur motivation rend l'accompagnement plus aisé, et ce faisant, l'autonomisation plus facile. Elle est ensuite contrariée par les obstacles à l'accès à l'emploi sur lesquels le dispositif n'a pas de prise. Les freins qui entravent le parcours des jeunes dans le dispositif, voire les écartent du marché du travail dans lequel le dispositif a pourtant ses entrées, assimilent la fin de l'accompagnement à une " défaite » personnelle, dont l'amertume croît en fonction de l'étiolement à la sortie de l'autonomie acquise à l'EPIDE ${ }^{19}$. Enfin, elle est une obstruction à l'accompagnement quand les jeunes sont « souverains " avant d'entrer dans le dispositif, et qu' ils rechignent à changer leurs manières de faire et leur façon d'être. L'encadrement est trop strict à leur goût, et la discipline nourrit le sentiment d'être dépossédés de leur subjectivité. Dans ce cas, la forme militaire est ressentie de manière ambivalente car elle les prive de leur quant-à-soi au nom même de l'autonomie. Ainsi, Brandon explique "faire son lit, ranger sa chambre et tout ça, franchement j'ai pas besoin qu'on me le dise. Je le fais déjà. Avec mon père forain, on allait de ville en ville avec la caravane, on vivait et on travaillait comme ça. Maintenant, je sais qu'ily a des trucs qui me manquent, que je ne connais pas. Donc c'est pour ça que je suis là. J'aimerais bien réapprendre des choses, parce que c'est intéressant. Comme le français, à parler des trucs, c'est pas évident déjà. Prononcer les bons mots. Les gens, ils rigolent un peu, mais c'est notre parler à nous... Mon projet, c'est d'être mécanicien dans l'armée de terre. À la base, j'aime bien la mécanique. L'EPIDE m'aiderait au niveau du sport, à faire mes tests physiques, à avoir une attitude plus posée on va dire, parce que c'est vrai, c'est pas très évident chez nous d'être calmes, on parle fort. Quand je suis arrivé là, ça m'a fait drôle. C'est calme, c'est carré. Mais pour le reste, j’ai pas besoin. Maintenant, j’ai ma caravane à moi, mon père fait sa vie de son côté. Je fais tout, les fêtes, les foires, les vendanges, le tirage de bois après les vignes. Je fais les pommes aussi. Et puis des fois, le travail par-derrière comme on dit, la ferraille quoi " (Brandon, 23 ans, père forain, a arrêté l'école en $5^{\mathrm{e}}$ ). Pour les jeunes déjà autonomes et indépendants avant

19. Sans qu'on puisse quantifier avec précision la proportion des jeunes concernés, ils font partie des $55 \%$ de jeunes qui sont en "sorties négatives " (pourcentage fourni par l'EPIDE, calculé sur la base des entrants à une période donnée qui n'accèdent ni à une formation ni à un emploi plus ou moins durable à la fin de l'accompagnement). 
d'entrer à l'EPIDE, la discipline provoque des tensions que la perspective d'un profit sur le plan social ou sur le plan professionnel peut calmer.

Le tiers environ des jeunes de notre échantillon dénonce la face sombre de l'EPIDE. Cette critique vient de jeunes qui soit disposent de ressources communautaires (à l'instar de Brandon) ou culturelles plus élevées que les autres, soit à l'inverse sont fortement préoccupés par des problèmes quotidiens de "survie » causés par une vulnérabilité extrême (à la limite de l'errance). Tous dénoncent une discipline trop forte, des abus d'autorité, voire des violences verbales à huis $\operatorname{clos}^{20}$.

Mais pour critiquable qu'il soit, le dispositif prend sa part au débat sur la socialisation des jeunes (quels qu'ils soient) comme le résultat d'un accompagnement strictement organisé et d'une formalisation codifiée des règles. Même si la formation est à rebours de l'individualisation (comme à l'E2C), elle a des effets sur la subjectivation des jeunes puisqu'ils y "font leur trou » et en utilisent les ressources. Leur autonomie, préexistante au dispositif ou construite grâce à lui, les préserve du sentiment de dépossession de leur marge d'action et de privation de leurs choix.

C'est l'ambivalence de la forme militaire : les jeunes subissent la contrainte, mais la réclament pour retrouver un rythme de vie que la situation de décrochage a cassé, plus trivialement s'occuper de soi sans l'aide d'un tiers : "Il fallait que j'entre à l'EPIDE parce que je commençais à péter les plombs, à faire n’importe quoi. J'avais envie de partir et tout... Ma mère, elle arrêtait pas de gueuler sur moi, j’en avais rien à foutre. Il fallait que je me fais reprendre, recadrer quoi » (Dylan, 20 ans, vit avec sa mère, a arrêté en BEP plomberie, veut devenir chauffeur routier). L'accompagnement est donc redevable des ajustements de la forme militaire à la volonté des jeunes de faire ce que l'EPIDE leur demande. Autrement dit, l'EPIDE ne s'adresse qu’à des jeunes déjà engagés dans un processus de changement et préalablement convaincus que la discipline, qui " recadre ", est le moyen de sortir de l'incertitude dans laquelle les cantonne l'absence de diplôme.

Si les jeunes sont réceptifs à la forme militaire et du même coup aux domaines de compétences et aux contenus transmis, cela est dû au travail sur le temps engagé avant le recours au dispositif, et qui se prolonge dans le dispositif : "Je tournais en rond, jallais péter les plombs " précise Sébastien (20 ans, vit chez ses parents, le père est peintre en bâtiment, il a arrêté en seconde générale, veut devenir horticulteur) quand Djamila (19 ans, vit chez ses parents, son père est au chômage et sa mère fait vivre le foyer grâce à des ménages, elle a arrêté en BEP Carrières sanitaires et sociales, et veut devenir gendarme) rappelle qu'elle passait son temps à s'occuper de ses frères et de ses sœurs, faire la cuisine et le ménage : "c'était ça tous les jours. À force, j’en ai eu marre". Les jeunes éprouvent (ou retrouvent), par la forme militaire, une expérience temporelle totale (Bessin, ibid.), c'est-à-dire une confi-

20. De ce point de vue, le dispositif n'est pas différent d'un cloître dont la nature n'est ni parfaite ni idéale (Durkheim, 1894 (1988)). 
guration qui substitue un temps linéaire et prévisible au temps vide et circulaire du passé (Zaffran, Vollet, 2018).

\section{Conclusion}

L'oxymore de la photo 1 , en début d'article (J'ai rejoint les rangs pour sortir du lot) n'est pas dépourvu de sens si l'on y voit le rappel du programme durkheimien d'éducation. Ce programme, d'une part, assimile la liberté individuelle au résultat socialisé d'une formation morale, d'autre part, associe la discipline avec des principes supérieurs offrant une perspective aux jeunes qui, pour ainsi dire, font les choses de leur propre chef dès lors qu'ils voient la règle comme désirable. À ce titre, la forme militaire de l'EPIDE est une incarnation de ce programme puisqu'elle poursuit un objectif d'autonomie sociale des jeunes sans diplôme. Toutefois, la socialisation par la forme militaire est-elle réductible à un redressement des corps par des procédures disciplinaires et des obligations de faire ce que le dispositif commande ? Autrement dit, l'EPIDE est-il un dispositif de contrôle social d'un État régulateur qui chercherait à transformer les jeunes en objet politique par des stratégies d'investissement des corps? On peut en douter car si on observe, à l'EPIDE, une organisation précise du temps et de l'espace et si on y croise des jeunes qui plient leurs conduites aux règles du dispositif et qui travaillent à redresser leur corps, leur engagement dans le dispositif procède d'un consentement éclairé. Par ailleurs, et en dépit des contraintes sociales et économiques qui pèsent sur leur parcours, ils sont libres d'y entrer, et libres d'en sortir ${ }^{21}$. Si les jeunes sans diplôme sont disposés à se plier aux règles du dispositif, c'est qu'ils le voient sous l'angle vertueux des avantages objectifs à moyen terme (une probable insertion professionnelle) et des ressources subjectives à court terme (une confiance en soi, une rigueur et une discipline). À la suite de quoi ils s'autonomisent en intériorisant les principes de la " socialisation épidienne " qui les transforme par des procédures transparentes et rationnelles, et qui sont d'autant plus efficaces que les jeunes eux-mêmes croient, ou finissent par croire, à cette fiction militaire.

Mélange de prestige et d'autorité traditionnellement attribués à l'armée (Merton \& Kitt, 1950), cette fiction transforme les jeunes en individus autonomes par leur croyance dans le mérite de l'engagement et l'égale reconnaissance, en dépit de leurs attributs sociaux ou ethniques, et malgré leur passé scolaire. Ainsi, l'EPIDE est assimilable à une institution car il assume une vocation éducative et un travail, sinon de conversion, du moins de transformation des jeunes (Denecheau \& Houdeville, 2018). Il crée de la cohésion (les entretiens insistent beaucoup sur l'entraide et l'attachement aux valeurs qu'incarne l'expression, souvent citée, " ne jamais laisser quelqu'un sur le bord de la route "), mais aussi parce qu'il accapare une part du temps et des intérêts des jeunes, puis les confronte dans un lieu dédié

21. Certains partent (environ $30 \%$ selon les chiffres communiqués) avant le terme de la formation, ce qui atteste que le dispositif n'est ni infaillible, ni que les jeunes sont totalement privés d'une capacité d'action. Parmi les raisons des abandons des jeunes évoquées par les formateurs, les désillusions et la critique ouverte du dispositif reviennent souvent. 
au modèle normatif d'adultes sûrs d'eux et assumant leurs rôles sociaux (Dubet, 2002). De plus, la forme militaire favorise l'exaltation de l'effort grâce auquel leur classement se fonde sur une logique différente du classement scolaire qu'ils ont connu (Menger, 2018). À ce titre, la force de la forme militaire est d'être moins une machine à rendre docile les corps qu'une fabrique de subjectivité par la reconnaissance des efforts et du mérite des jeunes dont la valeur personnelle n'est pas indexable sur le titre scolaire.

L'enquête et les résultats produits souffrent d'un certain nombre de limites. D'abord, nous n’avons pas interviewé des « déserteurs ». Il faut avouer qu'il est compliqué de les retrouver. Un entretien avec eux aurait permis d'éclairer différemment nos résultats. Ensuite, nous n'avons pas procédé à une comparaison systématique avec des jeunes sans diplôme ayant eu recours à un autre dispositif. Enfin, nous n'avons interviewé que les volontaires présents dans le dispositif. Cela nous a privé d'entretiens avec les jeunes ayant terminé leur formation, ce qui aurait permis de savoir si la contrainte exercée par le dispositif a produit un système d'autodiscipline suffisamment fort pour perdurer longtemps après la formation, et suffisamment tenace pour imprégner toute la structure de la personnalité. Sur ce point, on peut en douter pour deux raisons. La première est que l'EPIDE est une institution sans être totale. Le dispositif est irréductible à une caserne au sens militaire du terme et les jeunes n'y mènent pas une vie recluse. La seconde est l'irréductibilité de la forme militaire à une technique de mortification visant la privation, dans un lieu coupé du monde, des rôles de la vie courante Goffman, 1968) ou à une fonction de " réforme des habitus " (Mauger, $2001)^{22}$. Son emprise n'est jamais complète, et les jeunes peuvent toujours s'écarter du rôle que l'EPIDE leur assigne. Par ailleurs, l'accompagnement n'humilie pas, ne dépersonnalise pas et ne viole pas l'espace d'intimité, comme c'est le cas dans les organisations fermées.

Il reste que l'EPIDE réorganise l'expérience des jeunes sans diplôme. D’une part, il réengage des éléments de leur socialisation antérieure dans une nouvelle communauté qui cristallise des éléments identitaires ; d'autre part, il les coupe du monde extérieur le temps d'une formation ${ }^{23}$ dont les modalités sont minutieusement réglées. Loin d'être rebutés par les codes et les symboles militaires, les jeunes ${ }^{24}$ les assument, allant même jusqu’à affirmer leur « vocation épidienne » après avoir réclamé la fonction instituante d'un programme fai-

22. De ce point de vue, l'insertion ne serait qu'une «orthopédie morale » visant la transformation des propriétés morales, sociales et professionnelles des jeunes ainsi que leur acquisition des propriétés comportementales et des dispositions éthiques adaptées aux besoins et à l'idéologie du monde du travail (Mauger, ibid., p. 14).

23. Cette formation se fait en deux temps. Le premier, que nous venons de décrire, et le second, qui recentre le jeune sur son projet professionnel. Ce deuxième temps contribue sans doute à diluer les effets du premier. 24. Comme on l'a dit, tous les jeunes n'adhèrent pas ou peu à la forme militaire, ce qui serait une raison de leur " désertion ». Or, le départ prématuré est causé aussi par un niveau de vulnérabilité trop important pour assumer les contraintes de l'accompagnement. Nous avons assisté de façon fortuite au départ prématuré d'une jeune fille, et un échange rapide avec elle nous apprend que la principale raison de sa défection est le coût du transport hebdomadaire et l'accumulation des amendes pour défaut de titre de transport, mais aussi, comme elle le dit, le fait de ne "pas avoir la tête à ça pour le moment». 
sant défaut ailleurs. Ce programme régule l'action, propose des buts et des moyens, incarne des valeurs et des normes. Il assure aussi une fonction narrative par le récit de soi dans une mise en scène des actes héroïques, sous couvert d'emblèmes à forte charge affective et éthique. Dans une société où l'incertitude assimile les transitions biographiques à une épreuve, la force de la forme est d'être la scène où le jeune sans diplôme peut mener pour un temps, mais jusqu’à la victoire, les batailles contre soi.

\section{Bibliographie}

Allouche A. (2017), La Société du concours. L'empire des classements scolaires, Paris, Seuil.

Barrère A. (2011), L'Éducation buissonnière, Paris, Armand Colin.

Barrère A. (2013), "La montée des dispositifs : un nouvel âge de l'organisation scolaire ", Carrefours de l'éducation, 2013/2 (n³6), pp. 95-116.

Beaud S. (2018), La France des Belhoumi. Portraits de famille (1977-2017), Paris, La Découverte.

Bessin M. (2002), "Le recours au rite : l'exemple du service militaire ", Agora débats/jeunesses, $\mathrm{n}^{\circ} 28$, pp. 34-45

Denecheau B. \& Houdeville G. (dir.) (2018), « Le retour en formation : une vraie chance ? ", Formation Emploi, $\mathrm{n}^{\circ} 143$, octobre.

Dubar C. (Ed.) (1987), L'autre jeunesse. Des jeunes sans diplômes dans un dispositif de socialisation. Lille, Presses universitaires de Lille.

Dubet F. (2002), Le déclin de l'institution, Paris, Seuil.

Dubet F. (2009, Le travail des sociétés, Paris, Seuil.

Dubet F. (2010), «Déclin de l'institution et/ou néolibéralisme? ", Éducation et sociétés, n 25 , pp. 17-34.

Dubet F. (2018), Trois jeunesses. La révolte, la galère, l'émeute, Lormont, Le Bord de l'eau,

Durkheim E. (1992) [1902-1903], L'éducation morale, Paris, PUF.

Durkheim E. (1988) [1894], Les règles de la méthode sociologique, Paris, Champs Flammarion.

Goffman E. (1968), Asiles. Études sur la condition sociale des malades mentaux, Paris, Éditions de Minuit.

Hatto R., Tomescu-Hatto O., Muxel A. (2011), Enquête sur les jeunes et les armées : images, intérêt et attentes, Paris, Institut de recherche stratégique de l'École militaire (IRSEM). Disponible sur : http://www.irsem.defense.gouv.fr/IMG/pdf/etude_10.pdf

Keck F. (2012), «Goffman, Durkheim et les rites de la vie quotidienne», Archives de Philosophie, 2012/3 (Tome 75), pp. 471-492. 
Lacouette Fougère C., Lascoumes P. (2013), « Lévaluation : un marronnier de l'action gouvernementale?", Revue française d'administration publique, 2013/4, n 148, pp. 859-875.

Léger J.-F. (2005), L'évolution du rapport à l'autorité des militaires du rang in Gresle F. (dir.), Sociologie du milieu militaire. Les conséquences de la professionnalisation sur les armées et l'identité militaire, Paris, L'Harmattan.

Léon M.-H. (1999), Uniformes et formations. Socialisations et rites d'interaction dans un poste de commandement régimentaire, Paris, L'Harmattan.

Lévy-Garboua L. (1976), «Les demandes de l'étudiant ou les contradictions de l'université de masse ", Revue française de sociologie, 17-1, pp. 53-80.

Maillard D., Rouaud P. (2018), «"Demain, c’est loin". Transitions socioprofessionnelles des jeunes non diplômés ", Cereq Essentiels $\mathrm{n}^{\circ}$ 1, 20 ans d'insertion professionnelle des jeunes : entre permanences et évolutions, pp. 97-102.

Mauger G. (2001), « Les politiques d'insertion. Une contribution paradoxale à la déstabilisation du marché du travail », Actes de la Recherche en Sciences Sociales, vol. 136-137, pp. 5-14.

Menger P.M., (dir.) (2018), Le talent en débat, Paris, PUF.

Merton R. K. \& Kitt A. (1950), Contribution to the theory of reference group behavior in Merton R. K. \& Lazarfeld P. (eds.), Continuities in social research: studies in the scope and method of "the American soldier", New York, Free Press, pp. 40-105.

Pirone F. \& Rayou P. (2012), « Nouveaux internes, anciens décrocheurs : de l'évolution de la forme scolaire ", Revue française de pédagogie, 179, Avril-Juin, pp. 49-62.

Pirone F. (2015), Les " intermédiaires scolaires ", Micro-lycées et nouvelles frontières, in Rayou P. (coord.), Aux frontières de l'école. Institutions, acteurs et objets, Vincennes, Presses universitaires de Vincennes, pp. 133-154.

Schochet P. Z., Burghardt J., Mc Connell S. (2008), "Does the Job Corps work? Impact indings from the National Job Corps study", American economic review, vol. 98, $\mathrm{n}^{\circ} 5$, pp. 1864-1886.

Vincent, G. (dir.) (1994), L'éducation prisonnière de la forme scolaire. Scolarisation et socialisation dans les sociétés industrielles, Lyon, Presses Universitaires de Lyon.

Weber C. (2012), A genou les hommes ... Debout les officiers. La socialisation des Saint-Cyriens, Rennes, Pur.

Zaffran J. (2015), "Raccrocher et s'accrocher à un internat d'inspiration militaire. Le cas des décrocheurs scolaires qui s'engagent à l'Epide ", Revue française de sociologie, n $56-2$, pp. 331-356.

Zaffran J., Vollet J. (2018), Zadig après l'école. Pourquoi les décrocheurs scolaires raccrochent-ils?, Lormont, Le Bord de L'eau. 
